\title{
Impacto de la Sobretasa Arancelaria Implementada en el 2015 sobre el Nivel de Importaciones y el Pleno Empleo en el Ecuador
}

\section{Impact of the Tariff Surcharge Implemented in 2015 on the Level of Imports and Full Employment in Ecuador}

Jorge Izaguirre Olmedo

Evelyn Devenny Bazurto Campuzano

Cristina Plúa Muñoz

Universidad Internacional del Ecuador, Ecuador

Autor para correspondencia: joizaguirreol@uide.edu.ec; evbazurtoca@uide.edu.ec; mapluamu@uide.edu.ec Fecha de recepción: 10 de abril del 2019 - Fecha de aceptación: 27 de agosto del 2019

Resumen: Este estudio tiene por objeto determinar el impacto que tuvo la sobretasa arancelaria impuesta por el gobierno a través de la Resolución 11-2015, sobre las importaciones y el pleno empleo en el Ecuador. Para su desarrollo se obtuvieron datos del nivel de importaciones y el porcentaje de pleno empleo desde el 2007 hasta el 2018, con frecuencia anual. Para determinar el efecto en el pleno empleo se planteó un modelo econométrico que incluyó como variables explicativas a las importaciones, la tendencia y variables ficticias que permiten estimar el efecto de la regulación analizada. Los resultados indican que cada reducción porcentual de las importaciones afecta negativamente a la variación porcentual de pleno empleo en $0.38 \%$, por lo que el efecto de la reducción de importaciones fue una pérdida aproximada de 304,742 puestos de empleo pleno.

Palabras Clave: restricciones arancelarias; importaciones; pleno empleo; modelo de regresión lineal

\begin{abstract}
The purpose of this study is to determine the impact of the tariff surcharge imposed by the government through Resolution 11-2015, on imports and full employment in Ecuador. For its development, data were obtained on the level of imports and the percentage of full employment from 2007 to 2018, with an annual frequency. To determine the effect on full employment, an econometric model was proposed which included independent variables such as imports, trend and dummies variables that allow to estimate the effect of the analyzed regulation. The results show that each percentage reduction in imports negatively affects the percentage variation of full employment at $0.38 \%$, so that the effect of the reduction in imports was an approximate loss of 304,742 full employment positions.
\end{abstract}

Key Words: tariff restrictions; imports; full employment; linear regression model 


\section{Introducción}

Las restricciones a la importación son barreras comerciales al comercio internacional adoptadas por los gobiernos como medidas para disminuir el déficit de la balanza comercial, proteger la industria local, disminuir la salida de divisas, entre otros. Las estrategias que implementan los gobiernos para restringir el ingreso de productos extranjeros son la aplicación de barreras arancelarias como el incremento en el impuesto ad-valorem, los recargos específicos o la aplicación de ambos, además de las medidas no arancelarias como cupos de importación, prohibiciones y documentos de control. Estas barreras son reguladas internacionalmente a través de la Organización Mundial del Comercio.

Desde el año 2012, el gobierno ecuatoriano implementó medidas restrictivas a las importaciones con el objetivo de equilibrar la balanza de pagos. Estas restricciones se iniciaron con la aplicación de las Resoluciones 66-2012 y 67-2012 del Comité de Comercio Exterior COMEX, para restringir las importaciones de vehículos y celulares, respectivamente, mediante la asignación de cupos anuales para unidades y valores FOB. En el año 2013 se implementó la Resolución 116-2013 del COMEX para reformar la aplicación del Certificado de Reconocimiento INEN, según la Resolución y el Instituto Ecuatoriano de Normalización. Por último, en marzo del 2015, se implementó la Resolución 11-2015 que aplicó una sobretasa al impuesto Ad-valorem a más de 2,000 partidas arancelarias. Este estudio analiza el impacto de esta última resolución sobre las importaciones y el pleno empleo en el Ecuador, el mismo que se refiere a la situación de empleados que cumplan con 40 horas laboradas a la semana y un ingreso mayor o igual al salario mínimo mensual.

Un estudio realizado por la Cámara de Comercio de Guayaquil (2018) determinó que las medidas antes mencionadas no discriminaron las importaciones de Bienes de Capital, por lo tanto, estas restricciones tuvieron repercusión sobre el factor productivo. Considerando dicho resultado, es importante analizar el impacto que pudieron tener las restricciones sobre diferentes variables del sector real a fin de que puedan tomarse en consideración para posibles medidas económicas futuras.

Como punto de partida del análisis, la Tabla 1 muestra la evolución de la tasa de pleno empleo y de las importaciones en miles de dólares. Así mismo, presenta los años en los que se expidieron las resoluciones que buscaban limitar la salida de divisas y equilibrar la balanza de pagos del país.

Tabla 1.

Pleno Empleo, Importaciones y Resoluciones Comex

\begin{tabular}{|c|c|c|c|}
\hline Periodo & $\begin{array}{l}\text { Empleo Adecuado/Pleno } \\
\text { (\%) (Total nacional) }\end{array}$ & $\begin{array}{l}\text { Importaciones (Miles de } \\
\text { dólares FOB) }\end{array}$ & Resoluciones COMEX \\
\hline Dic-07 & 43,2 & $12.895 .241,1$ & \\
\hline Dic-08 & 44,8 & $17.551 .929,7$ & \\
\hline Dic-09 & 39,2 & $14.071 .455,4$ & \\
\hline Dic-10 & 44,7 & $19.278 .714,0$ & \\
\hline Dic-11 & 45,5 & $23.088 .117,0$ & \\
\hline
\end{tabular}




\begin{tabular}{|llll}
\hline Periodo & $\begin{array}{l}\text { Empleo Adecuado/Pleno } \\
(\%) \text { (Total nacional) }\end{array}$ & $\begin{array}{l}\text { Importaciones (Miles de } \\
\text { dólares FOB) }\end{array}$ & Resoluciones COMEX \\
\hline Dic-12 & 46,5 & $24.181 .570,2$ & $\begin{array}{l}\text { Resolución 66-2012: Restricción } \\
\text { cuantitativa anual de importación para } \\
\text { vehículos. } \\
\text { Resolución 67-2012: Restricción } \\
\text { cuantitativa anual para la importación de } \\
\text { teléfonos celulares }\end{array}$ \\
\hline Dic-13 & 47,9 & $\begin{array}{l}\text { Resolución 116-2013: Certificado de } \\
\text { reconocimiento INEN }\end{array}$ \\
\hline Dic-14 & 49,3 & $25.763 .812,4$ & $\begin{array}{l}\text { Resolución 11-2015: Sobretasa } \\
\text { arancelaria a las importaciones }\end{array}$ \\
\hline Dic-15 & 46,5 & $26.421 .483,7$ & $\begin{array}{l}\text { Resolución 49-2015, 50-2015: Cuota } \\
\text { global para la importación de teléfonos } \\
\text { celulares }\end{array}$ \\
\hline Dic-16 & 41,2 & $20.446 .797,9$ & \\
\hline Dic-17 & 42,3 & $15.545 .157,4$ & \\
\hline Dic-18 & 40,6 & $19.031 .064,0$ & \\
\hline & & $22.118 .882,7$ & \\
\hline
\end{tabular}

Fuente: Banco Central del Ecuador (2014, 2019), INEC (2019), COMEX.

Como puede observarse en la tabla, las importaciones crecieron sobre los 20 millones de dólares FOB en el año 2011 hasta el año 2014 donde registró 26 millones de dólares. En el año 2015 tuvo una caída de 6 millones con relación al año anterior y en el año 2016 continuó decreciendo en 5 millones hasta llegar a 15.5 millones de dólares FOB. A partir del año 2017 se observa una recuperación de la serie. La Figura 1 ilustra la evolución de las importaciones.

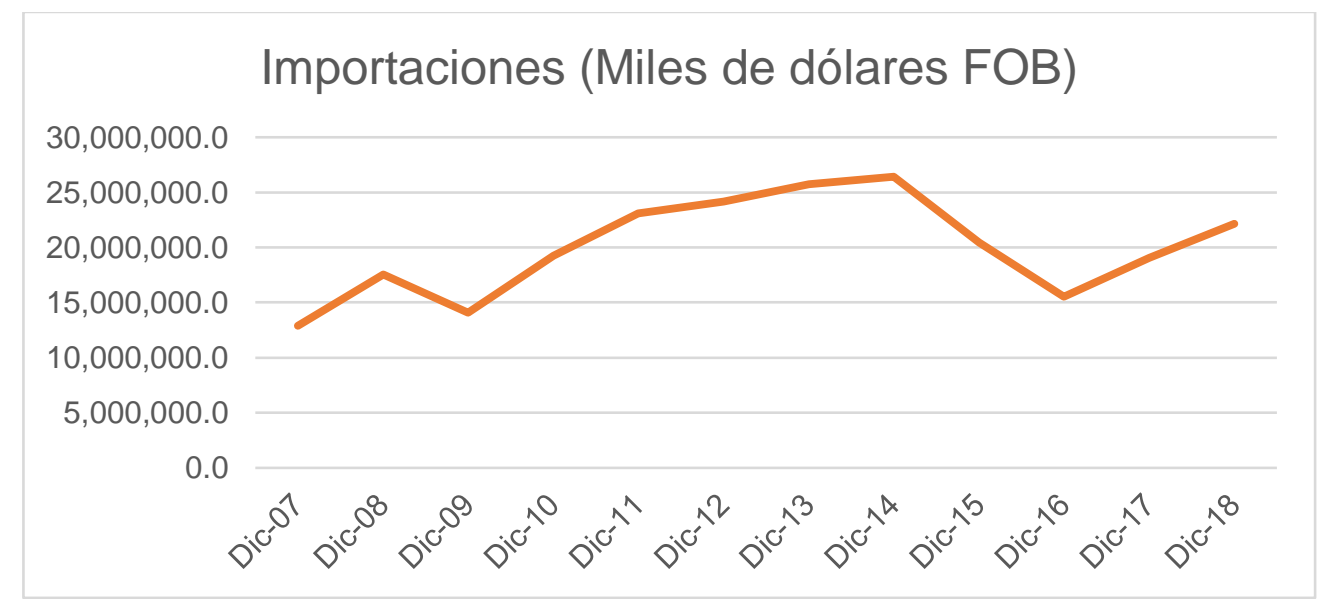

Figura 1. Importaciones (Miles de dólares FOB).

Elaboración propia. Fuente: Banco Central del Ecuador (2014, 2019)

Por otra parte, la tasa nacional de Pleno Empleo tuvo un crecimiento constante desde el 2009 hasta el 2014, en que registró el 49.3\%. En el año 2015 la tasa de empleo pleno bajó a 46,5\% y para el año 2018 la tasa nacional se registró en 40,6\%. Esta caída pudo tener múltiples factores. Lo que se desea determinar en este estudio es la magnitud de la caída registrada en el 2015 que corresponda a la disminución de las importaciones debido a la implementación de la Resolución 11-2015. 


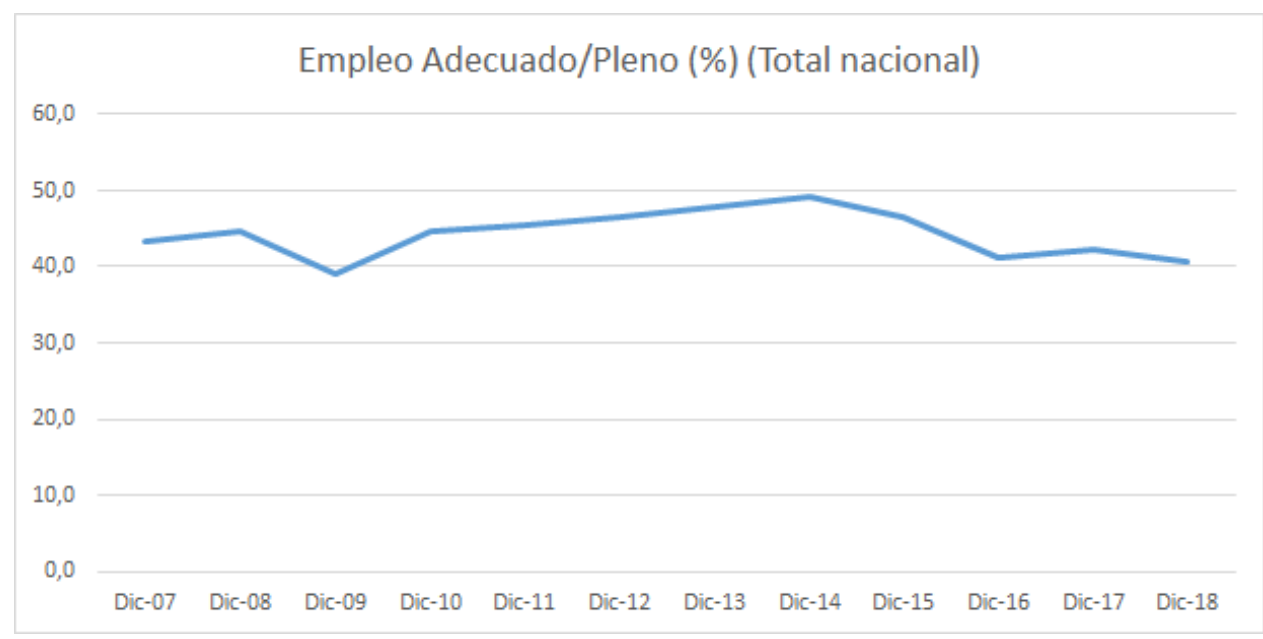

Figura 2. Empleo Adecuado/Pleno.

Elaboración propia. Fuente: INEC, (2019).

En base a lo anterior, el objetivo general de este estudio es determinar el impacto que tuvo la sobretasa arancelaria impuesta por el gobierno a través de la Resolución 11-2015, sobre las importaciones y el pleno empleo en el Ecuador. Para la consecución del mismo, se han planteado tres objetivos específicos: i) Determinar el efecto de la resolución 11-2015 sobre el nivel de importaciones en el Ecuador; ii) Plantear un modelo econométrico para estimar el efecto de la resolución 11-2015 y la caída de las importaciones sobre la tasa de pleno empleo en el Ecuador; y, iii) Calcular el efecto de las restricciones arancelarias sobre la tasa de pleno empleo.

\section{Metodología}

La información con la que se realizó el estudio fue obtenida de las estadísticas de las importaciones en miles de dólares FOB publicadas por el Banco Central del Ecuador. La tasa nacional del Pleno Empleo o Empleo Adecuado se obtuvo del Instituto Ecuatoriano de Estadísticas y Censos. Las Resoluciones se obtuvieron del Comex y se considera para este estudio la Resolución 11-2015. Tanto las series de nivel de importaciones como de tasa de pleno empleo fueron trabajadas en variaciones porcentuales.

Para determinar si el efecto de la Resolución 11-2015 sobre el nivel de pleno empleo es significativo, se desarrolló una regresión lineal múltiple estimada a través de mínimos cuadrados ordinarios, en base al siguiente modelo:

$$
P E_{t}=f\left(M_{t}, d, d t r e n d, \text { trend, } P E_{t-1}\right)
$$

$P E$ denota la variación porcentual del nivel de pleno empleo y constituye la variable dependiente del modelo. El modelo es autorregresivo de orden 1. $M$ representa la variación porcentual de las importaciones, $d$ es una variable ficticia que toma el valor de 1 a partir del 2015 en el que se aprobó la resolución y 0 para cualquier otro año; dtrend representa una variable ficticia iterativa que toma el valor 0 antes del 2015 y el valor de la tendencia a partir de dicho año; trend es la tendencia de $P E$. 


\section{Revisión de la Literatura}

Hill (2015), menciona que las reformas arancelarias son instrumentos en las políticas comerciales adoptadas por los gobiernos. Desde la creación de la OMC, como consecuencia de varios tratados multinacionales en 1995, se promueve el comercio libre limitando a los gobiernos a adoptar políticas que restrinjan las importaciones y eliminar las barreras a libre tránsito de las mercancías. Por otra parte, los gobiernos adoptan medidas para restringir el libre comercio con el objetivo de conservar las fuentes de trabajo nacional, proteger las industrias importantes para la seguridad nacional, proteger a los consumidores de productos peligrosos y fortalecer los objetivos de comercio exterior. Los aranceles, que es un impuesto a las importaciones pueden ser ad-valorem, específicos, mientras que las barreras no arancelarias se establecen mediante la asignación de cupos de importación, prohibiciones o documentos de control.

Arévalo, Pastrano y González (2016) desarrollaron un análisis económico - financiero de las empresas importadoras de Ecuador, luego de la implementación de reformas arancelarias por parte de gobierno ecuatoriano como medida de mejora a los saldos registrados en Balanza de Pagos. Para su análisis, los autores consideraron las reformas desde el 2007 hasta el 2015. El estudio se desarrolló en base a un trabajo de campo considerando el caso de tres empresas importadoras en el país. Los resultados señalan problemas de capital de trabajo debido a los costos adicionales que debían pagarse al Servicio Nacional de Aduanas, adicional a ello, el nivel de importaciones disminuye lo que colocaba a estas empresas en categorías más bajas frente a sus productores en el exterior, generando como consecuencia un menor plazo de crédito y afectando a la liquidez de las mismas.

Paguay (2016) estudió el efecto de las salvaguardas en los mercados ecuatorianos en el período 2010 - 2015. En su análisis observó que las medidas proteccionistas impuestas por el gobierno pudieron favorecer a sectores como el textil y el de bebidas alcohólicas, afectando también en forma negativa a ciertos sectores como el automotriz. Como conclusión de su estudio, destaca el éxito que tuvo el gobierno en la reducción del déficit de balanza comercial, señalando también que existen materias primas básicas para la producción que mantienen una sobretasa. Al respecto de esta última afirmación y en un ámbito más reciente, la Cámara de Comercio de Guayaquil (2018) analizó el impacto de las salvaguardas sobre las importaciones de los bienes de capital y nivel de producción en el Ecuador. Para la realización del estudio se planteó un modelo econométrico que tomaba como variable dependiente a las importaciones de bienes de capital y como variables independientes al tipo de cambio real, ingreso nacional y salvaguardas. Los resultados mostraron que la reducción en la importación de bienes de capital fue de un $22 \%$, lo que implicó una pérdida en la producción no petrolera del $0.8 \%$, lo que implica $\$ 725$ millones de menor producción anual.

Analizando otra perspectiva de los efectos de las salvaguardas, Caza y Yacelga (2016) estudiaron los efectos de la Resolución 11-2015 del COMEX en importaciones, exportaciones, recaudación por salvaguardas y empresas que realizan comercio exterior vía terrestre con Colombia. El estudio consideró una muestra del $70 \%$ del total de importaciones y exportaciones y arrojó como resultado una reducción del déficit de balanza comercial con Colombia en un $21.8 \%$, una recaudación en salvaguardas por 65.3 millones de dólares. Sin embargo, las cifras 
muestran que, aunque se consiga una reducción del déficit, las exportaciones también se ven afectadas por la medida y el efecto es $18 \%$.

Por otra parte, Pico y Coello (2017) analizaron la restricción de importaciones en el sector automotriz del Ecuador y su impacto económico. Para su análisis, consideraron el período de 2010 al 2015 y abordaron tres tipos de restricciones: cupos de importación, salvaguardas y recargos específicos. El estudio fue de tipo exploratorio y se basó en información secundaria recopilada del Sistema Nacional de Aduanas, la Asociación de Empresas Automotrices del Ecuador - AEADE y el Sistema Nacional de Información - SNI. Como conclusión de su análisis, destacan la importancia que tuvo la implementación de barreras arancelarias para limitar la salida de divisas del país, en una situación económica adversa. Señalan también que el cambio de la matriz productiva pudo mejorar a algunos sectores, limitando así el efecto que pudo producir la limitación de exportaciones. No obstante, los resultados encontrados no son concluyentes debido al tipo de estudio que fue desarrollado; efecto que desea medirse en esta investigación.

Hidalgo (2018) analizó las políticas arancelarias implementadas en Ecuador entre el 2007 y el 2016 y su impacto en la producción del sector manufacturero. Para el efecto, el autor planteó un modelo econométrico que tomaba como variable dependiente al PIB per cápita del sector manufacturero y como variables independientes a la tendencia, el PIB nacional per cápita y variables ficticias para los períodos en los que se aplicaron las medidas. Los resultados encontrados mostraron un impacto negativo de las medidas en el nivel del PIB per cápita del sector manufacturero. No obstante, este resultado no se ha considerado como válido para la elaboración de este artículo debido a que el estudio de Hidalgo (2018) utilizó el PIB nacional per cápita para explicar el PIB manufacturero per cápita, pudiendo incurrir en un error de doble causalidad; lo que anularía los resultados obtenidos.

Finalmente, Soria (2018) realizó un análisis econométrico del efecto de las salvaguardas en las importaciones del Ecuador en período 2014 - 2016. Para ello, planteó un modelo de regresión múltiple estimado a través de mínimos cuadrados ordinarios en el que tomó como variable dependiente el nivel de importaciones y tomó como variables independientes a las salvaguardas, FODINFA e IVA. Los resultados de su estudio señalan que un incremento en el $1 \%$ en el nivel de salvaguardas, generan una reducción del $0.0005 \%$ del nivel de importaciones. Sin embargo, las series no fueron tratadas como series temporales, por lo que las conclusiones no fueron consideradas para el diseño de la metodología del presente estudio.

\section{Resultados}

Luego de la revisión independiente de las variables de pleno empleo e importaciones que se realizó en la introducción del estudio, se procedió a la estimación del modelo econométrico. A continuación se presentan las estadísticas de la regresión: 
Tabla 2.

Estadísticas de la regresión

\begin{tabular}{ll}
\hline Estadístico & Valor \\
\hline Coeficiente de correlación múltiple & 0,990 \\
Coeficiente de determinación $\mathrm{R}^{\wedge} 2$ & 0,981 \\
$\mathrm{R}^{\wedge} 2$ ajustado & 0,957 \\
Error típico & 0,016 \\
ANOVA (valor crítico F) & 0,002 \\
Observaciones & 10 \\
\hline
\end{tabular}

Acorde a lo presentado en la tabla, se puede observar en el valor crítico de la $F$ que el modelo es significativo y explica a la variable dependiente. Así mismo, se observar que el coeficiente de determinación asciende a 0.98 , lo cual implica un buen ajuste del modelo. A continuación se presentan los coeficientes de las variables independientes y su nivel de significancia.

Tabla 3.

Modelo de regresión

\begin{tabular}{lll}
\hline Variables Independientes & Coeficientes & Probabilidad \\
\hline Intercepción & $-0,069$ & 0,021 \\
Variación porcentual importaciones & 0,375 & 0,001 \\
Dummy 2015 - 2017 & 0,434 & 0,010 \\
Dummy iterativa 2015-2017 & $-0,056$ & 0,005 \\
Tendencia & 0,013 & 0,027 \\
$\operatorname{Ar}(1)$ & $-0,178$ & 0,092 \\
\hline
\end{tabular}

Como puede observarse, la variación porcentual de las importaciones, la tendencia y las variables ficticias son significativas. La variable de autorregresión es significativa al 10\%. Los coeficientes confirman la hipótesis previa planteada en el estudio. El coeficiente de la variación porcentual de importaciones indica que existe una relación directa entre esta variable y la variación porcentual del pleno empleo, por tanto, al disminuir el crecimiento de las importaciones se afecta negativamente al crecimiento del pleno empleo. Específicamente, por cada reducción del $1 \%$ en las importaciones, la variación porcentual del pleno empleo se reduce en $0.38 \%$.

El efecto de las importaciones sobre el pleno empleo tiene particular importancia debido a las consecuencias en términos de consumo que esta representa. Una reducción del pleno empleo implica una reducción en el nivel de ingreso de los hogares, lo cual se traduce en una disminución del consumo. Una reducción del consumo implica una desaceleración de la economía, lo cual podría implicar un aumento en el nivel de desempleo de empresas que no se encuentren relacionadas al sector de comercio exterior.

La tendencia es positiva lo que implica un crecimiento de la serie. La variable ficticia indica que a partir del 2015 el promedio de la serie es mayor que antes del mencionado año. Este coeficiente estaría contradiciendo el resultado obtenido a través de la variación porcentual de 
importaciones, sin embargo, el análisis se complementa con el coeficiente de la variable ficticia iterativa. Esta última tiene coeficiente negativo, por tanto, a pesar de que en promedio la serie sea mayor luego de la resolución, ésta va disminuyendo con cada año transcurrido. A continuación, se presenta el gráfico de la serie y su estimación.

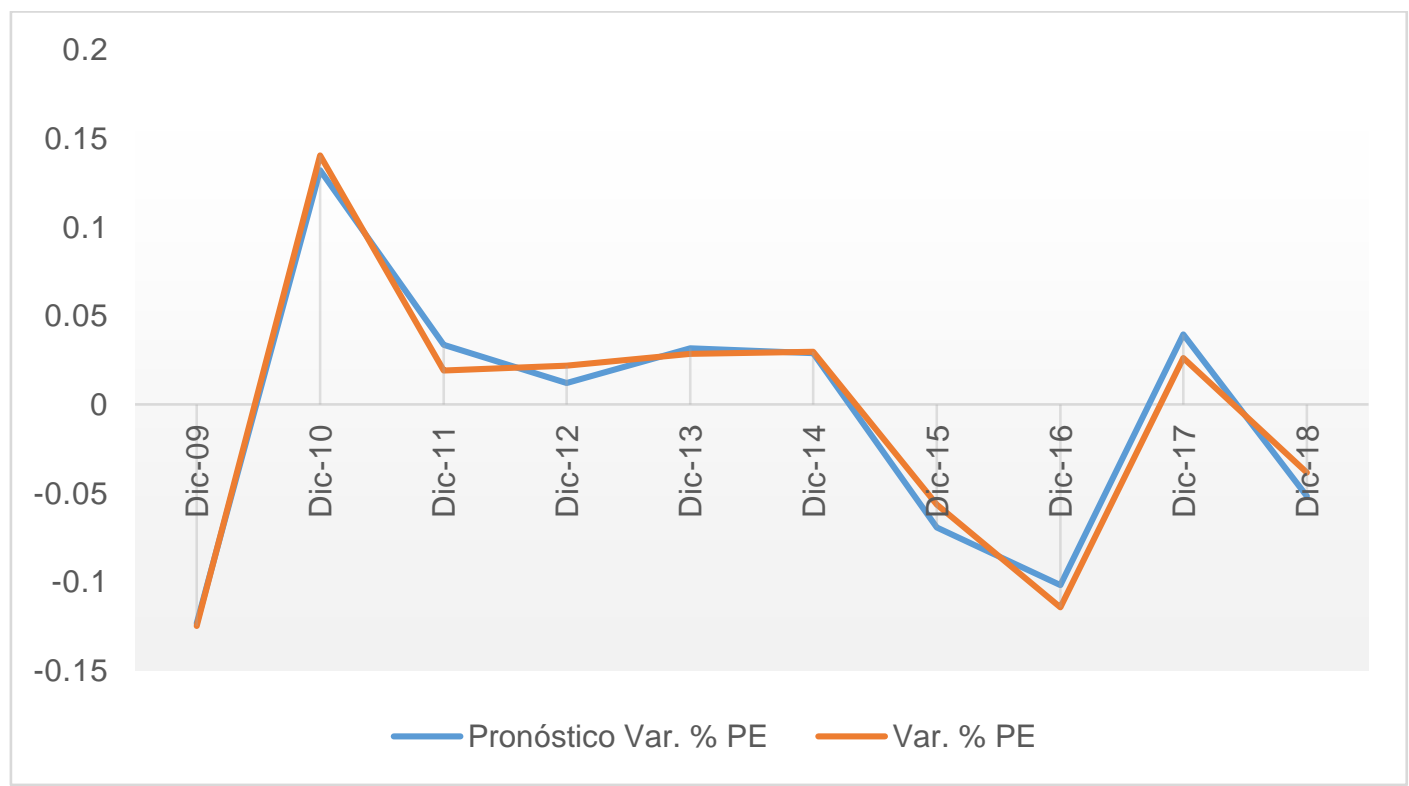

Figura 3. Variación porcentual del Empleo Adecuado/Pleno y su pronóstico.

\section{Conclusiones}

Una vez presentados los resultados de la estimación, se concluye que la Resolución 11 2015 tuvo el impacto esperado sobre las importaciones, el cual era conseguir la reducción de su nivel de manera inmediata; sin embargo, los datos muestran que la afectación de dichas importaciones también impactó negativamente en la tasa de pleno empleo o empleo adecuado. El monto estimado de puestos de pleno empleo perdidos asciende a 304,742 por causa de la disminución de las importaciones, sin perjuicio de otros efectos positivos o negativos que puedan haber afectado a la serie debido a otras variables.

La evidencia sugiere que las regulaciones implementadas por el gobierno ecuatoriano, más allá de tener la intención de equilibrar la balanza de pagos, afectaron negativamente al sector real. Una pérdida de puestos de pleno empleo podría significar una reducción del nivel de consumo de la economía, lo que implicaría una pérdida del ingreso real. La recomendación en términos de políticas públicas sería analizar los efectos colaterales que pueden tener las medidas restrictivas para salvaguardar que la solución implementada no tienda a crear un problema paralelo. De igual manera, se recomienda el desarrollo de nuevos estudios que relacionen el impacto en términos de consumo e inflación, debido a variaciones en importaciones por restricciones comerciales. 


\section{Bibliografía}

Arévalo, K., Pastrano, E., \& González, C. (2016). Análisis económico-financiero ante las reformas arancelarias. Caso de estudio: empresas importadoras de Ecuador. Revista Publicando, 3(7), 476 - 491.

Banco Central del Ecuador. (2016). Boletín anuario 38. Importaciones por secciones de la nomenclatura aduanera.

Banco Central del Ecuador. (2019). Estadísticas del Sector Externo. Importaciones FOB mensuales por continente, área económica y país de procedencia.

Cámara de Comercio de Guayaquil. (2018). El impacto de las salvaguardias sobre la producción en el Ecuador.

Cámara de Comercio de Guayaquil. (2018). INEC publica cifras de empleo a marzo del 2018. Informe de Posición Estratégica $N^{\circ} 250$.

Castillo, R., \& Moncayo, J. (2015). Empleo y Condición de Actividad en el Ecuador. Revista de Estadísticas y Metodologías del INEC, Volumen 1, 29 - 54.

Caza, J., \& Yacelga, C. (2016). Análisis de los efectos de la aplicación de las salvaguardias en las importaciones y exportaciones en el intercambio comercial por carretera Ecuador Colombia. Sathiri $n^{o} 11,42$ - 60.

De-Gregorio, J. (2007). Macroeconomía: Teoría y Políticas. Ira. edición.

González, M. (Mayo de 2016). El Efecto de las Salvaguardias sobre la Importación de Bienes de Capital y la Producción. Obtenido de http://economiaenjeep.blogspot.com/2016/05/elefecto-de-las-salvaguardiassobre-sobre-la.html

Hidalgo, A. (2018). Análisis del Impacto de las Políticas Arancelarias en el Sector Económico Manufacturero del Ecuador en el período 2007-2016. Quito: Universidad Central del Ecuador.

Hill, C. (2015). Negocios Internacionales. Competencia en el Mercado Global. México: McGrawHill/Interamericana Editores S.A.

Instituto Nacional de Estadísticas y Censo. (2019). Encuesta nacional de Empleo, Desempleo y Subempleo .

Paguay, D. (2016). Impacto de las salvaguardias a las importaciones en el Ecuador período 2010 - 2015. Guayaquil: Universidad de Guayaquil. Facultad de Ciencias Económicas.

Pico, L., \& Coello, R. (2017). Análisis de la restricción a las importaciones del sector automotriz en el Ecuador periodo 2010 - 2015 y su impacto en la economía. INNOVA Research Journal 2017 Vol 2, No. 1, 128 - 142.

Rodríguez Guevara, D., \& González Uribe, G. (2017). Principios de Econometría. Medellin: Intituto Tecnológico Metropolitano.

Soria, N. (2018). Evaluacion y analisis econometrico del efecto de las salvaguardias en las importaciones del Ecuador, periodo 2014-2016. Quito: Universidad Central del Ecuador. 